\title{
A 1.5g SMA-actuated Microglider looking for the Light
}

\author{
Mirko Kovač, André Guignard, Jean-Daniel Nicoud ${ }^{\star}$, Jean-Christophe Zufferey, Dario Floreano \\ Ecole Polytechnique Fédérale de Lausanne (EPFL) \\ Laboratory of Intelligent Systems (LIS, http://lis.epfl.ch) \\ CH-1015 Lausanne, Switzerland \\ ${ }^{\star}$ DIDEL SA (http://www.didel.com) \\ \{mirko.kovac, andre.guignard, jean-christophe.zufferey, dario.floreano\}@epfl.ch, nicoud@ didel.com
}

\begin{abstract}
Unpowered flight can be used in microrobotics to overcome ground obstacles and to increase the traveling distance per energy unit. In order to explore the potential of goal-directed gliding in the domain of miniature robotics, we developed a $22 \mathrm{~cm}$ microglider weighing a mere $1.5 \mathrm{~g}$ and flying at around $1.5 \mathrm{~m} / \mathrm{s}$. It is equipped with sensors and electronics to achieve phototaxis, which can be seen as a minimal level of control autonomy. A novel 0.2g Shape Memory Alloy (SMA) actuator for steering control has been specifically designed and integrated to keep the overall weight as low as possible. In order to characterize autonomous operation of this robot, we developed an experimental setup consisting of a launching device and a light source positioned $1 \mathrm{~m}$ below and $4 \mathrm{~m}$ away with varying angles with respect to the launching direction. Statistical analysis of 36 autonomous flights demonstrate its flight and phototaxis efficiency.
\end{abstract}

\section{INTRODUCTION}

In the animal kingdom, gliding is used to prolong jumps, escape predators and to rapidly reach a distant location, e.g. in locusts [1], gliding snakes [2] or certain species of ants [3]. Gliding may be a powerful behavior for robotic locomotion as well. It would allow microrobots to overcome ground obstacles or to cover relatively large distances in short periods of time. In this paper, we present the development and characterization of an ultra light microglider flying at a velocity of $1.5 \mathrm{~m} / \mathrm{s}$ (Fig. 1) as an initial step in the exploration of gliding as an alternative or complementary locomotion principle in miniature robotics.

To date, different attempts have been made to build small scale flying robots using rotors [4], fixed wings [5] or flapping wings [6], but none of these systems have been designed for unpowered flight. A remarkable $2.2 \mathrm{~g}$ microglider using a four-bar piezo actuator to steer has recently been presented [7]. Although this realization is a master piece of micromechatronics, no characterization of autonomous gliding has been provided so far. In addition, this glider flies at a velocity of more than $5 \mathrm{~m} / \mathrm{s}$ and requires a $8 \mathrm{~m}$ turning radius to perform a 180 degree turn [8].

In order to obtain efficient gliding distances with small scale systems, special care has to be taken to achieve very low weight because the lift to drag ratio is known to naturally decrease with size, due to increasing viscous and boundary layer effects [9], [10]. To achieve low weight and low power consumption, while maintaining an acceptable level of complexity allowing for fast prototyping, we opted for a

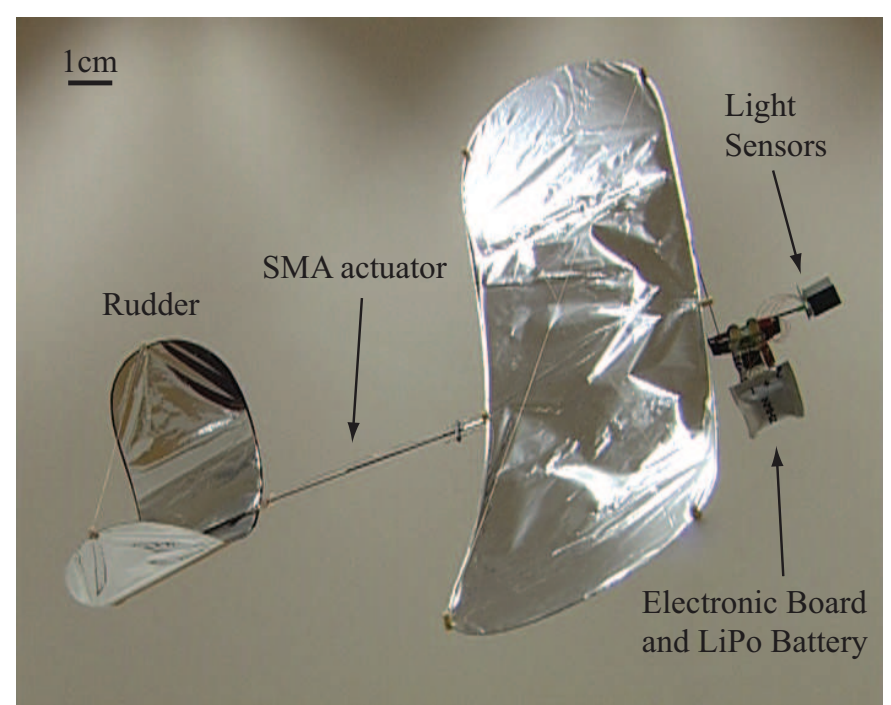

Fig. 1. 1.5g SMA-actuated microglider capable of autonomous phototaxis, wingspan $24 \mathrm{~cm}$, length $22 \mathrm{~cm}$, flying at around $1.5 \mathrm{~m} / \mathrm{s}$

relatively new kind of steering system. We developed a $0.2 \mathrm{~g}$ Shape Memory Alloy (SMA) actuator that is harmoniously integrated into the structure of the microglider and allows for direct control of the rudder.

Two tiny photoreceptors and a simple control strategy were used to provide our $1.5 \mathrm{~g}$ robot with a minimal level of autonomy. The microglider was fully tested and characterized for its gliding and phototaxis capabilities. Closeup views of the microglider and demonstrations of its behavior can be seen in the accompanying video and at http://lis.epfl.ch/jumpglider.

In the following sections we first present the construction principles of the microglider along with the design of the SMA actuator and the embedded electronics. We then describe the control mechanism enabling the phototaxis behavior. Finally, we report on the characterization procedure and flight results.

\section{MICROGLIDER}

\section{A. Airframe}

Our design strategy is aimed at low weight and simplicity, for fast prototyping. Therefore, the same airframe architecture (Fig. 2) used in our previous research activities in indoor flying robots [5], [10]-[12] has been chosen, 

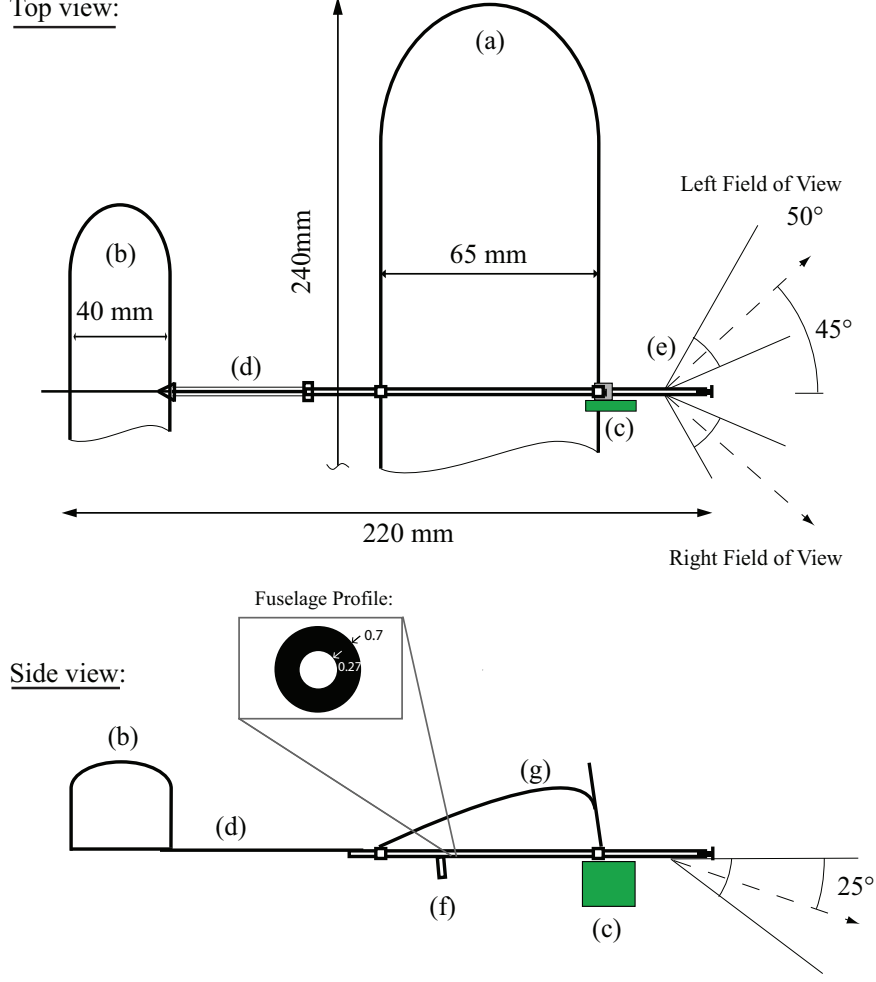

Fig. 2. Construction plan of the microglider. (a) main wing, (b) rudder, (c) Electronic Board and battery, (d) SMA actuator, (e) light sensors, (f) catcher for launching, glued to the bottom side of the fuselage, $(\mathrm{g})$ camber support.

but with particular effort to further reduce the weight and energy consumption. The construction principle is to use a structural frame of high strength for stability and to cover the aerodynamical surfaces with light and smooth materials for minimal aerodynamical friction. Carbon fiber material with a fiber volume fraction of $65 \%$ and a specific density of $1.55 \mathrm{~g} / \mathrm{cm}^{3}$ has been chosen for the fuselage and the frame of wing, elevator and rudder.

The fuselage presents the major contribution to the weight of the airframe (Table I). Therefore, a $0.7 \mathrm{~mm}$ carbon tube (Fig. 2, Fuselage Profile) has been used to provide strength while minimizing the mass.

The frame of wings and rudder has been constructed out of $0.3 \mathrm{~mm}$ round profile carbon rods to allow for homogeneous flexing and formation of the 3-dimensional wing structure. The material for the actual wing surface is biaxially-oriented polyethylene terephthalate (boPET) polyester film (trade name "Mylar foil") because of its high tensile strength and dimensional stability. Its weight of $6 \mathrm{~g} / \mathrm{m}^{2}$ allows for covering the elevator and main wing with a mass of only $0.09 \mathrm{~g}$. In addition, a camber support (Fig. 2 (f)) made of $0.12 \mathrm{~mm}$ thick carbon bars has been added in order to maintain structural stability of the main wing. The resulting weight of the airframe including wings is only $0.31 \mathrm{~g}$.

\section{B. Actuation}

Small airplanes flying at low velocities are strongly constrained by weight and power consumption. Different actua-
TABLE I

WEIGHT BUDGET

\begin{tabular}{|c||c|}
\hline Part & Mass $(\mathrm{g})$ \\
\hline Electronic Board & 0.33 \\
\hline Battery 10mAh & 0.55 \\
\hline Fuselage & 0.18 \\
\hline Front wing & 0.1 \\
\hline Rudder & 0.03 \\
\hline Light sensors & 0.1 \\
\hline SMA actuator & 0.2 \\
\hline Cables and soldering & 0.02 \\
\hline \hline Total mass & $\mathbf{1 . 5 1}$ \\
\hline
\end{tabular}
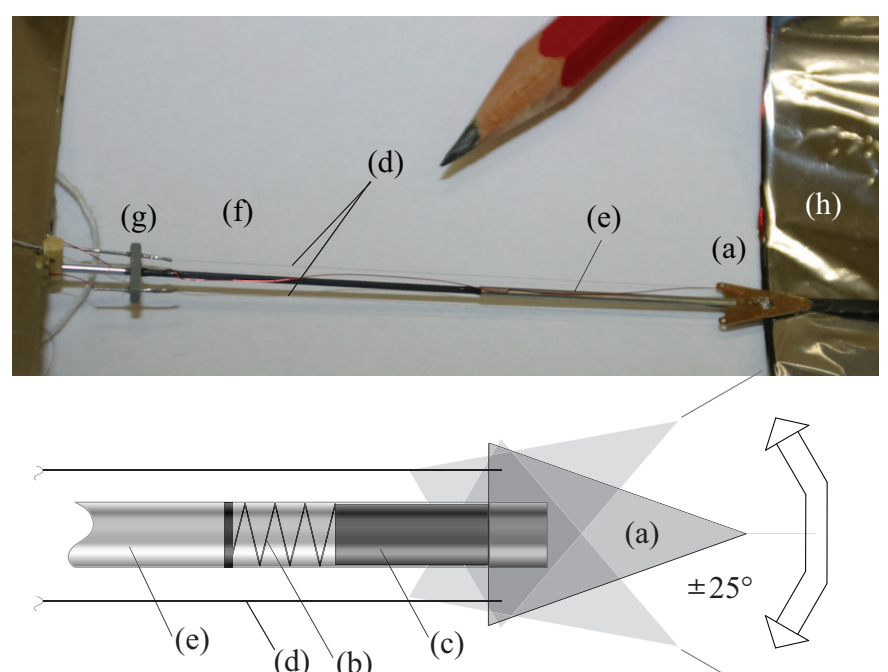

(d) (b)

Fig. 3. 0.2g SMA actuator, (a) horn, (b) spring, (c) piston, (d) SMA wire, (e) steel tube, (f) carbon fuselage, (g) frame with electrical interface to Electronic Board, (h) rudder.

tion systems could potentially be employed for actuating the control surfaces, such as magnetic coils, piezo actuators or SMA. Table II compares three examples of different actuator types used on airplanes of less than $10 \mathrm{~g}$. The mass and power consumption can easily be quantified. However, other important figures of merit like commercial availability, mechanical complexity and force output are rather qualitative.

Magnetic coils have the advantage of relatively uncomplicated manual assembly and can easily be obtained commercially, but deliver comparably lower forces and are difficult to control precisely in position. Piezo materials have very low power consumption, deliver high forces and repetition rates, but with very limited displacement. They usually require complex micromechanical design for its amplification [6] and adequate costly equipment and expertise to fabricate the actuators. In addition, the very low weight of the actuator itself is diluted with weight expensive drive electronics to achieve the high voltage that is required (200V in [7] leads to a 
TABLE II

ACTUATOR COMPARISON

\begin{tabular}{|c||c|c|c|c|c|c|}
\hline Actuator type & Mass $(\mathrm{g})$ & Drive Electronics $(\mathrm{g})$ & Power $(\mathrm{mW})$ & Commercial availability & Mechanical complexity & Force output \\
\hline \hline Magnetic coils [13] & 0.15 & 0.02 & 180 & +++ & + & - \\
\hline Piezo [7] & 0.05 & 0.2 & 7 & + & - & ++ \\
\hline SMA [14] & 0.12 & 0.01 & 171 & + & + & ++ \\
\hline
\end{tabular}

$(+++$ : very favorable, ---: very unfavorable)

weight of about $0.2 \mathrm{~g}$ for the DC-DC converter, its peripheral components and additional board material). SMA wires offer comparably large displacements of around $5 \%$ of their length [15], very high power density and are used alternatively to conventional magnetic coils in hobbyist remote control airplanes [14]. The drive electronics is minimal and consists of one $0.01 \mathrm{~g}$ transistor. However, potential disadvantages of SMA is a higher power consumption, lower repetition rate and more delicate dynamic control compared to, e.g., magnetic coils, due to hysteresis effects. For our application of rudder control, Nickel Titanium Alloy (Nitinol) wire, also known as "Artificial Muscles" [15], has been used due to its very high strength to weight ratio, precise control, simplicity and the very low power consumption (less than $160 \mathrm{~mW}$, depending on the duty cycle).

The working principle of SMA wire is that it exploits the crystallographic structure change of martensite to austenite (thermoelastic martensitic transformation) when heated above the transition temperature. This phase change produces a force that can be used for actuation. We used "Alloy M Flexinol ${ }^{\mathrm{TM}}$ " wire [16] with a transition temperature of $70^{\circ} \mathrm{C}$ and $25 \mu \mathrm{m}$ diameter for maximal performance and low power consumption.

The actuator we developed (Fig. 3) consists of (a) a Copper-Beryllium horn, (e) a $0.7 \mathrm{~mm}$ steel tube, $(\mathrm{g})$ a frame with electrical interface and (d) two $25 \mu \mathrm{m}$ Flexinol $^{\mathrm{TM}}$ wires attached to the frame and the horn. The stability of the actuator is given by the carbon fuselage (f). The wires are activated with a Pulse Width Modulation (PWM) signal as described below, which leads to a contraction of up to $3.5 \mathrm{~mm}$ with a maximal force of $0.069 \mathrm{~N}(\hat{=} 7 \mathrm{~g})$ at the attachment point of the horn. This leads to a deflection of the horn and the rudder which is glued on the horn. The point of rotation is the attachment point of the other SMA wire. Depending on the PWM duty cycle, a torque of up to $0.27 \mathrm{mNm}$ can be obtained. The counterpart of this movement is the custom made brass spring (b) with a spring constant of $45.8 \mathrm{~N} / \mathrm{m}$ which ensures back alignment of the rudder to the neutral position at zero PWM duty cycle.

\section{Perception}

Autonomous navigation requires a sensory system. Many different solutions are commercially available, but complex sensors such as Inertial Measurement Units (IMU) or GPS are far too heavy or energy consuming to be used on such a microglider. We decided therefore to equip the microglider with one photodetector on each side in order to demonstrate minimalist autonomous operation similar to the phototactic vehicles proposed by Braitenberg [17]. To this end, TAOS TSL237T High Sensitivity Light Sensors were chosen for their low weight of only $0.05 \mathrm{~g}$, low cost and sensitivity in the visual spectrum.

\section{Embedded Electronics}

A new 0.33g Electronic Board (EB) (Fig. 1) has been specifically designed for our microglider. This low weight EB incorporates a 14pin 8-Bit CMOS Microchip ${ }^{\mathrm{TM}}$ PIC16F676 microcontroller with a $20 \mathrm{MHz}$ oscillator. It supports low voltage (3V) power supply and possesses built-in analog to digital converters allowing different types of interfaces with the sensors and actuators. The EB also includes one Single Chip (SC70) MOSFET transistors to drive the SMA actuator.

\section{E. Control}

The microglider is intended to fly towards a light source. Depending on the difference between the two light sensors, it determines if the light source is on the left or right side of the microglider and the corresponding SMA wire is activated (Fig. 4) by a PWM signal of $196 \mathrm{~Hz}$ frequency.

The current sent to the wire is controlled by PWM and its duty cycle is calculated according to the fraction of the two values of light sensors as follows:

$$
\begin{aligned}
& \left.\begin{array}{l}
p_{l}=100 \cdot\left(1-\frac{s_{r}}{s_{l}}\right) \\
p_{r}=0
\end{array}\right\} \text { if } s_{r}<s_{l} \\
& \left.\begin{array}{l}
p_{l}=0 \\
p_{r}=100 \cdot\left(1-\frac{s_{l}}{s_{r}}\right)
\end{array}\right\} \text { if } s_{r}>s_{l}
\end{aligned}
$$

where $s_{r}$ and $s_{l}$ are the output values of the light sensors on the right and left side. $p_{r}$ and $p_{l}$ are the PWM duty cycle values sent to the SMA wire on the right and left side of the actuator. If the light source is in front of the glider, the rudder deflection is very little. However, if the light source is on one side, the ratio between the two sensory values is high and the resulting PWM duty cycle induces a stronger deflection of the rudder leading to a more pronounced direction change towards the light.

The actuator control has to be balanced with the response of the glider to rudder movements and its reaction time for perception. According to basic airplane dynamics, a too 


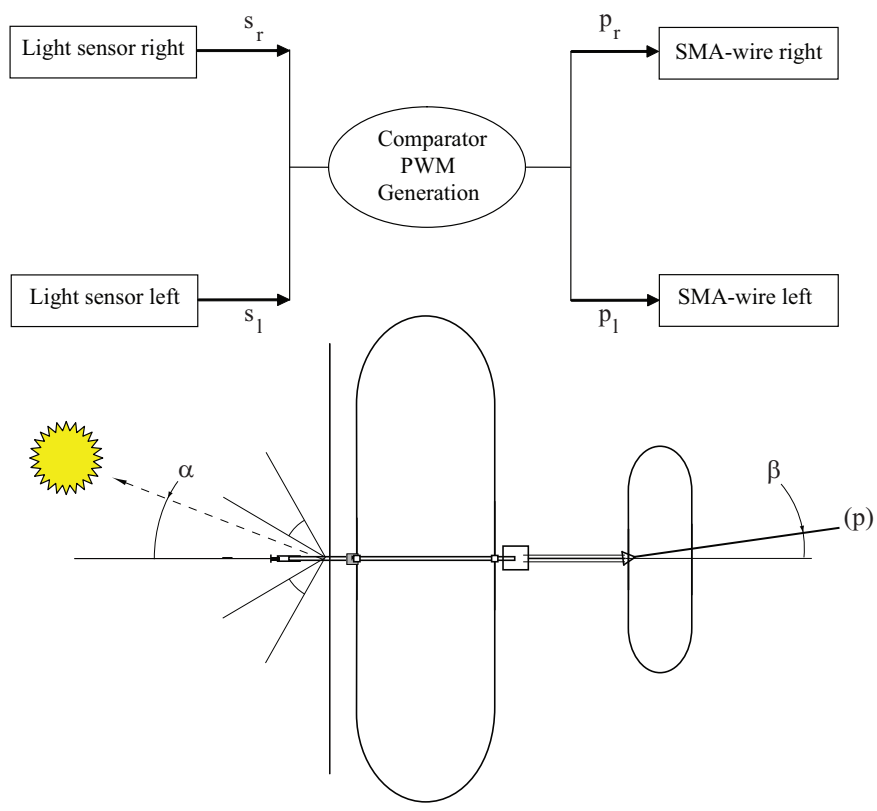

Fig. 4. Sensory actuator cycle scheme and characterisation setup. A 75W light source is positioned at $50 \mathrm{~cm}$ distance (6601x) with varying light source angle $\alpha$ to measure the deflection angle $\beta$ of the rudder (Fig. 5 and 6), (p) pointer for measurement of $\beta$

strong deflection leads to abrupt roll and sideways diving of the glider. In order to prevent this behavior, the maximal PWM duty cycle has been determined empirically to be 80 (corresponding to $80 \%$ high state of the PWM signal (1)), leading to a rudder deflection of $25^{\circ}$. In addition, an adjustment to the background light level has been implemented. Before the flight, the glider is calibrated by exposing it to the highest illumination difference that lead to the maximal ratio of the two sensor values in the particular environment. The maximal PWM duty cycle is then assigned to the maximal ratio of the two sensory values perceived.

\section{F. Control Characterisation}

In order to assess the quality of the control strategy and test the response to changes of light source location, we used a $75 \mathrm{~W}$ light bulb placed at $50 \mathrm{~cm}$ distance in front of the microglider with a varying angle $\alpha$ (Fig. 4)). The rudder deflection $\beta$ was then measured by means of a $10 \mathrm{~cm}$ pointer (Fig. 4 (p)) attached to the rudder. Figure 5 shows the output values of the two light sensors $s_{r}$ and $s_{l}$ and the PWM duty cycles $p_{r}$ and $p_{l}$ (according to (1)) versus the light source angle $\alpha$. The field of view of each sensor spans $50^{\circ}$ between $20^{\circ}$ and $70^{\circ}$ on each side (Fig. 2).

The rudder movement (Fig. 6) shows a deflection of up to $25^{\circ}$ to the side of the light source gradually adjusting to the light source position. This characterization indicates proper functioning of the control mechanism in the static

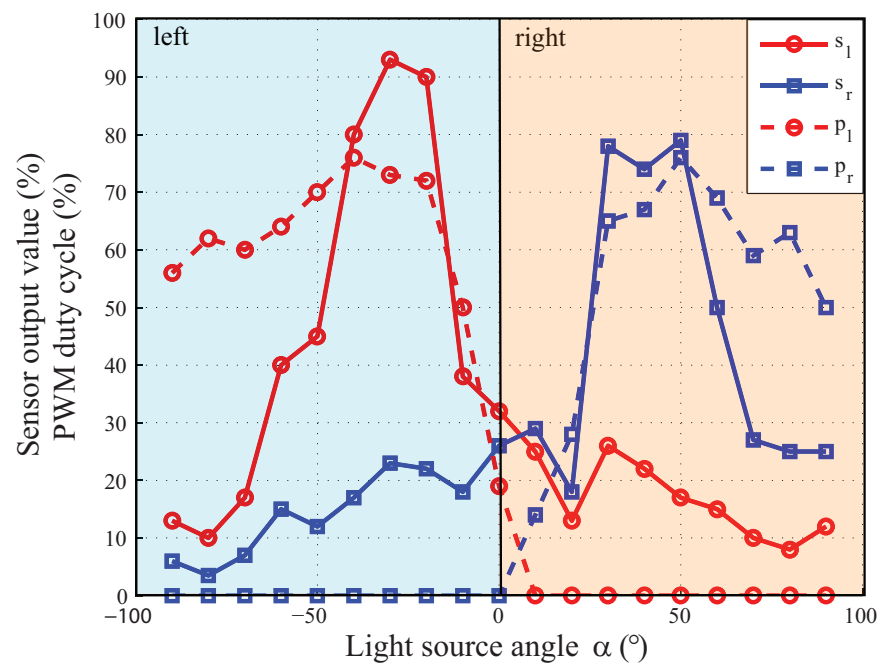

Fig. 5. Output values of the two light sensors $s_{l}$ and $s_{r}$ in percentage to saturation and PWM duty cycles $p_{l}$ and $p_{r}$ (according to (1)) versus the light source angle $\alpha$ (Fig 4).

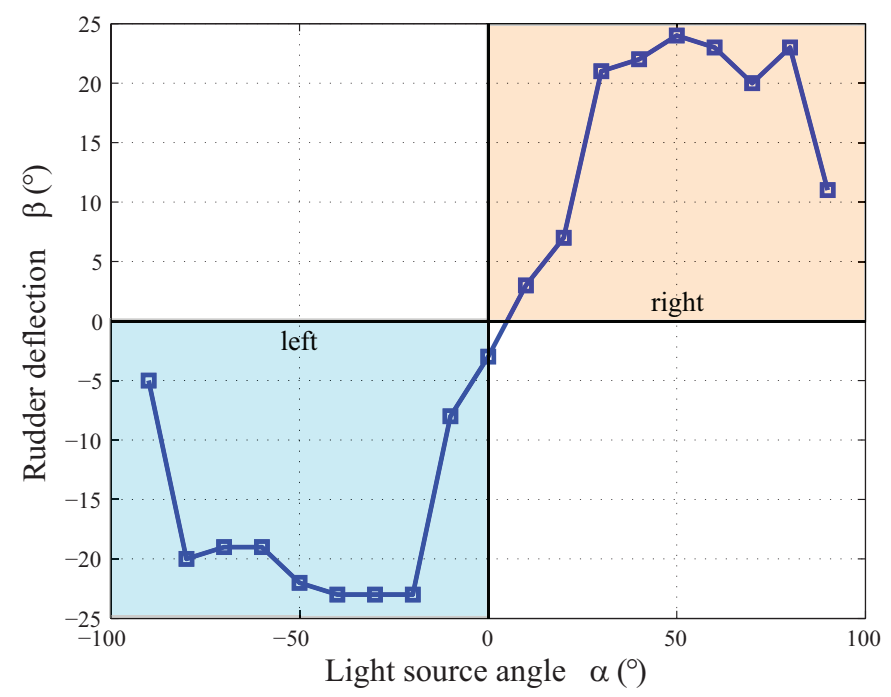

Fig. 6. Rudder deflection $\beta$ depending on light source angle $\alpha$ (Fig 4).

case. Further experiments were required to demonstrate this behavior in flight.

\section{IN FLIGHT EXPERIMENTS}

In order to characterize the flying abilities of the microglider, a number of systematic in flight experiments were carried out and statistically analyzed. These experiments aimed at testing whether the microglider is actually able to detect the direction of a light source and fly towards it autonomously.

\section{A. Launching Device}

A launching device (Fig. 7) has been built to minimize the error due to changes in launching conditions. It consists of a carbon tube attached to a spring made of synthetic gum. The microglider is prepared for launch by positioning the catcher (Fig. 2 (f)) to the tip of the carbon tube. By manually pulling 


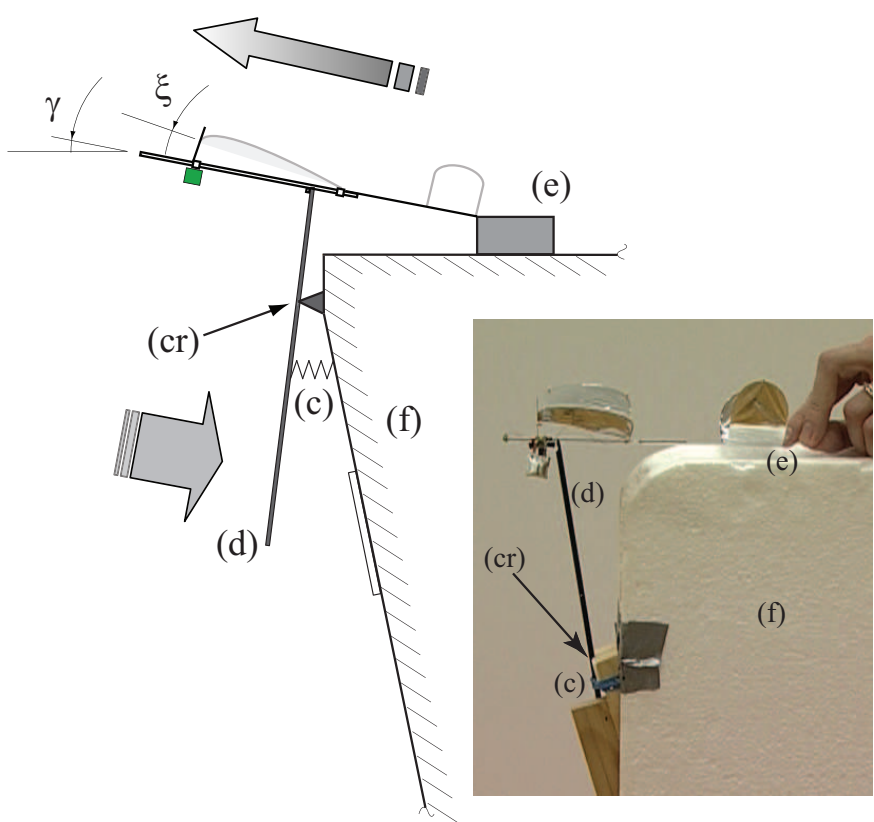

Fig. 7. Layout and picture of the launching device. $\gamma$ launching angle, $\xi$ angle of incidence, (c) spring, (d) carbon tube, (e) marker, (f) mounting, (cr) center of rotation. By pulling the glider backwards manually, the spring is charged and provides energy for the launching of the glider at a given launching angle.

the glider backwards against the marker, the spring is charged and provides the required energy for launch. The launching parameters, i.e. launching angle and launching velocity, can be systematically and precisely adjusted by changing the marker position.

\section{B. Aerodynamical Optimization}

In order to improve in flight operation of our microglider, we needed to optimize the flight distance per given launching altitude, usually referred to as gliding ratio (2).

$$
\text { Gliding ratio }=\frac{\text { Lift }}{\text { Drag }}=\frac{\text { Flight distance }}{\text { Launching hight }}
$$

The easiest way to influence the flight performance for a given launching setup is to change the angle of incidence $\xi$, i.e. angle between the wing and the fuselage (Fig. 7). In order to find the optimal angle of incidence, the microglider was launched with a velocity of $2 \mathrm{~m} / \mathrm{sec}$ and a fixed launching angle of $3.5^{\circ}$, whereby both values were measured optically using video and image processing (Fig. 7 and 9).

For each of the six different angles of incidence (Fig. 8), three launches were carried out from a height of $1 \mathrm{~m}$ and the flight distance was measured (Fig. 9). The optimal angle of incidence has been found to be $4.6^{\circ}$, corresponding to a maximal average gliding ratio of 5.63 (Fig. 8). This setting was used throughout the phototaxis experiments.

\section{Phototaxis}

We present a series of experiments where the glider was launched using the launching device to detect the direction of a light source and fly towards it. To this end, three series of

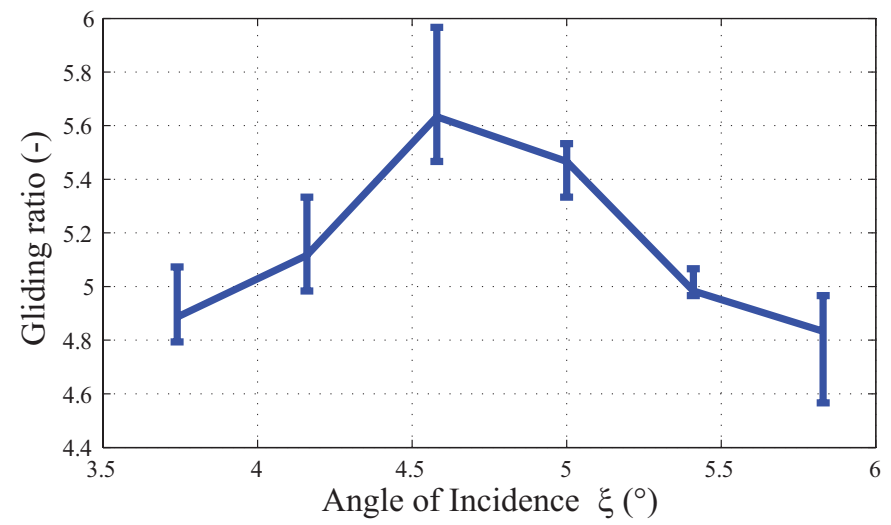

Fig. 8. Gliding ratio optimization. For every angle of incidence $\xi$, three subsequent launches have been carried out. Average values with error bars.
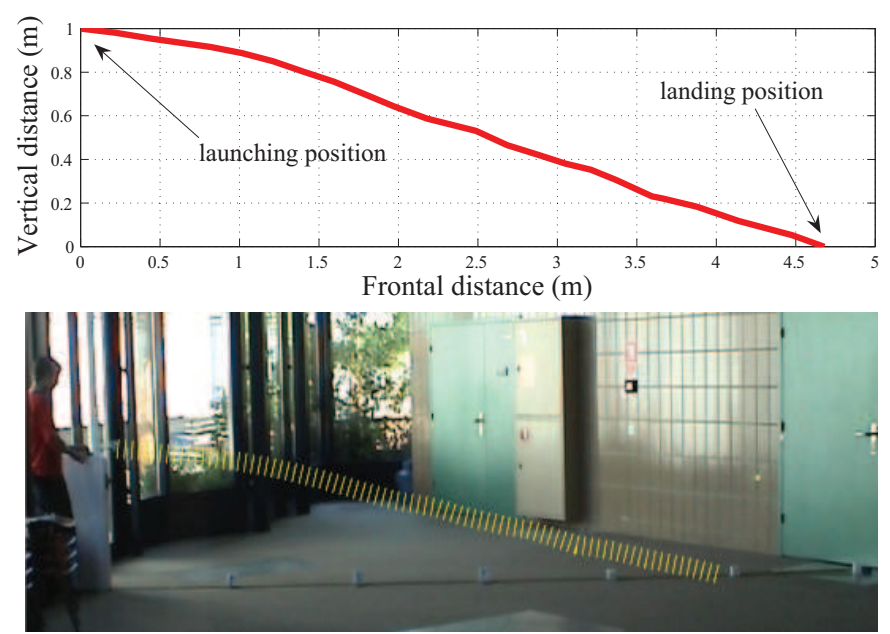

Fig. 9. Flight trajectory, launching velocity $2 \mathrm{~m} / \mathrm{sec}$ and launching angle $\gamma=3.5^{\circ}$.

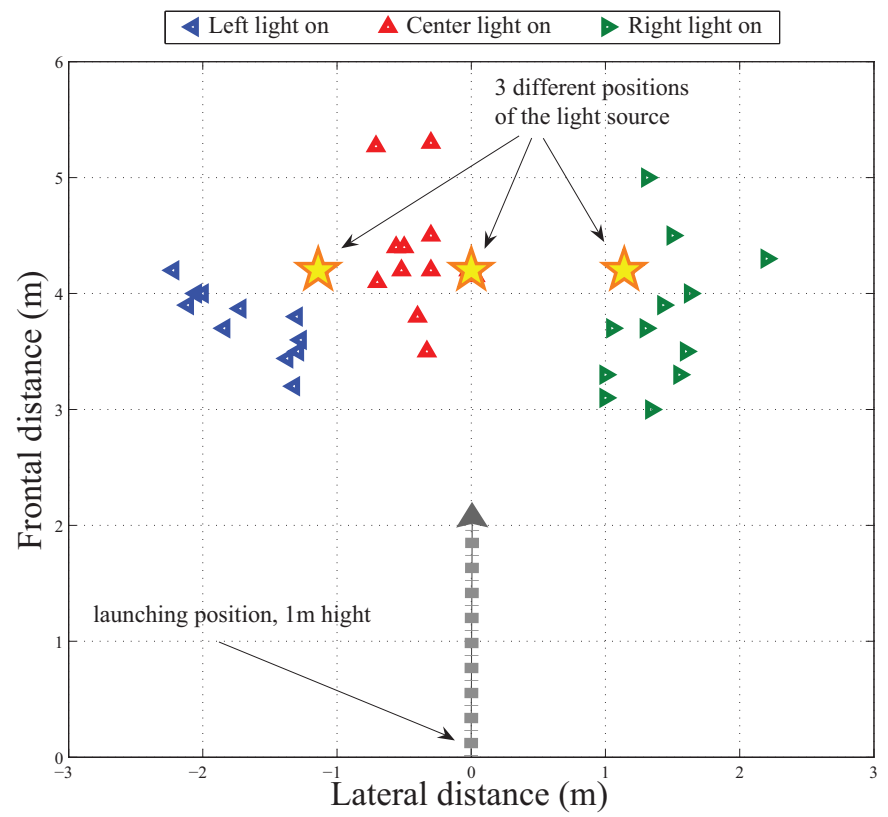

Fig. 10. Top view of the setup for the phototaxis experiments. Stars indicate the three possible light source locations. For every of the three locations, 12 subsequent launches have been carried out. The triangles mark the landing positions. 


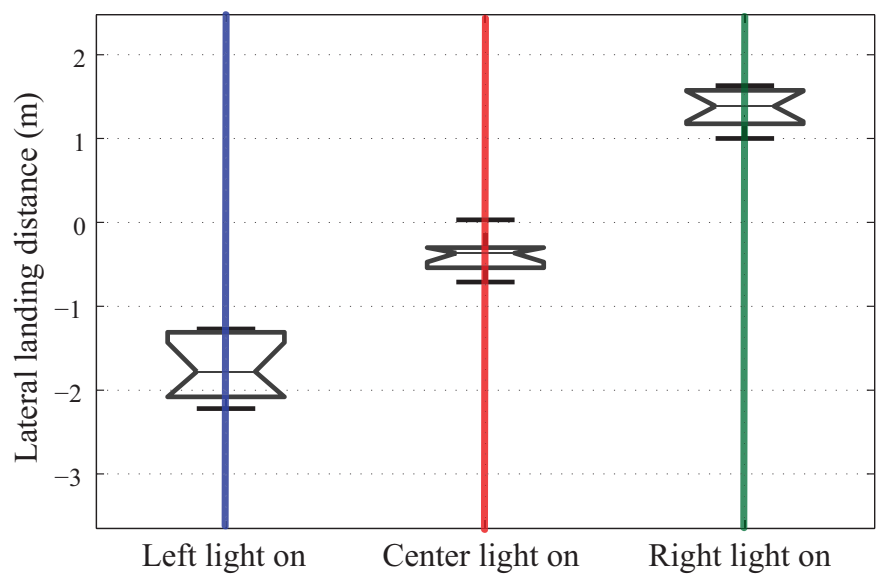

Fig. 11. Results of One-way ANOVA test on lateral distance of the landing points with respect to launching axis. The midline is the median, the borders of the box represent the upper and the lower quartile. The notches permit the assessment of the significance of the differences of the medians. When the notches of two boxes do not overlap, the corresponding medians are significantly different at the $95 \%$ confidence level.

launches were carried out, each with a different position of the light source (Fig. 10). For every light source position, the microglider was launched 12 times using the same launching parameters. The landing positions were measured and statistically analyzed. Due to time constrains these experiments have not been carried out with the latest, $1.5 \mathrm{~g}$ microglider, but with an earlier prototype weighting two more grams with the same dimensions, control characteristics and a $25 \%$ lower gliding ratio of 4.3 , flying at $1.7 \mathrm{~m} / \mathrm{s}$. However, preliminary tests with the $1.5 \mathrm{~g}$ version (see video attachment) show similar or improved behavior.

In order to test if the microglider actually displayed a phototactic behavior, a one-way analysis of variance (ANOVA) test [18] was carried out on the three sets of landing positions. In our case, ANOVA was used to test if the microglider behaved differently when the light source was at a different position. The statistical evaluation was based on the lateral landing distance with respect to the launching axis (Fig. 10).

For the three series of phototaxis experiments, the significance level $p$ of the ANOVA test has been found to be less than 0.0001 (Fig. 11). These experiments indicate that the microglider efficiently and reliably detects the light source and flies towards it.

\section{CONCLUSION AND OUTLOOK}

We developed an ultra light weight microglider and demonstrated its capability of autonomous phototaxis. In order to keep the overall weight as low as possible and explore the potential of SMA for rudder control, we developed and integrated a novel 0.2g SMA actuator. The control mechanism for the sensory-motor cycle was characterized in the static case and the angle of incidence optimized, in order to maximize the gliding ratio. 36-sample in flight phototaxis experiments were performed and statistically analyzed show- ing that the microglider efficiently and reliably detects the light source and flies towards it.

This microglider prototype is a first step in our exploration of gliding as an alternative or complementary locomotion for miniature robotics to overcome obstacles and increase the traveling distance per energy unit. The next steps will include the development of a folding mechanism for the wings and the integration of a terrestrial locomotion mode (e.g. jumping mechanism). Further weight reduction, bioinspired optimization of the aerodynamics and the usability of solar cells are also under investigation.

\section{ACKNOWLEDGMENTS}

We would like to thank Philippe Vosseler for the fabrication of the actuator components. This project is supported by the EPFL.

\section{REFERENCES}

[1] R. Santer, P. Simmons, and F. C. Rind, "Gliding Behaviour Elicited by Lateral Looming Stimuli in Flying Locusts," Journal of Comparative Physiology, vol. 191, no. 1, pp. 61-73, 2004.

[2] J. Socha and M. LaBarbera, "Effects of Size and Behavior on Aerial Performance of two Species of Flying Snakes (Chrysopelea)," The Journal of Experimental Biology, vol. 208, pp. 1835-1847, 2005.

[3] S. Yanoviak, R. Dudley, and M. Kaspari, "Directed Aerial Descent in Canopy Ants," Nature, vol. 433, pp. 624-626, 2005.

[4] I. Kroo and P. Kunz, "Mesoscale flight and miniature rotorcraft development," in Fixed and Flapping Wing Aerodynamics for Micro Air Vehicle Applications, ser. Progress in Astronautics and Aeronautics. AIAA, 2001, vol. 195, pp. 503-517.

[5] J.-C. Zufferey, A. Klaptocz, A. Beyeler, J.-D. Nicoud, and D. Floreano, "A 10-gram Microflyer for Vision-based Indoor Navigation," in IEEE/RSJ Int. Conf. on Intelligent Robots and Systems, Beijing, China, 2006.

[6] R. S. Fearing, K. H. Chiang, M. H. Dickinson, D. L. Pick, M. Sitti, and J. Yan, "Wing Transmission for a Micromechanical Flying Insect," in Proc. IEEE Int. Conf. on Robotics and Automation, vol. 2, 2000, pp. 1509-1516.

[7] R. J. Wood, S. Avadhanula, E. Steltz, M. Seeman, J. Entwistle, A. Bachrach, G. L. Barrows, S. Sanders, and R. S. Fearing, "Design, Fabrication and Initial Results of a $2 \mathrm{~g}$ Autonomous Glider," in IEEE Industrial Electronics Society 2005 Meeting, Raleigh North Carolina, 2005.

[8] J. P. Entwistle and R. S. Fearing, "Flight simulation of a 3 gram autonomous glider," Master's thesis, University of California, Berkeley.

[9] T. J. Mueller, Fixed and Flapping Wing Aerodynamics for Micro Air Vehicle Applications, ser. Progress in Astronautics and Aeronautics. AIAA, 2001, vol. 195.

[10] J.-D. Nicoud and J.-C. Zufferey, "Toward Indoor Flying Robots," in IEEE/RSJ Int. Conf. on Robots and Systems, Lausanne, Switzerland, 2002, pp. 787- 792 .

[11] J.-C. Zufferey and D. Floreano, "Fly-inspired Visual Steering of an Ultralight Indoor Aircraft," IEEE Transactions on Robotics, vol. 22, no. 1, pp. 137-146, 2006.

[12] J.-C. Zufferey, "Bio-inspired Vision-based Flying Robots," Ph.D. dissertation, Ecole Polytechnique Fédérale de Lausanne (EPFL), 2005.

[13] Micro flier radio. [Online]. Available: http://www.microflierradio.com

[14] M. Keennon, "Muscle Wire Technology for Micro and Indoor Models," in NEAT Micro Seminars 2004.

[15] J. Conrad and J. Mills, Stiquito Advanced Experiments with a Simple and Inexpensive Robot. IEEE Computer Society, 1997.

[16] Info-sheet No. 13, Nitinol Alloy Types, Conditions and Surfaces. [Online]. Available: http://www.memory-metalle.de

[17] V. Braitenberg, Vehicles - Experiments In Synthetic Psychology. Cambridge, MA: The MIT Press, 1984.

[18] R. Hogg and J. Ledolter, Engineering Statistics. Collier Macmillan London, 1987. 